\title{
Weak and strong convergence theorems for a finite family of non-Lipschitzian nonself mappings in Banach spaces
}

\author{
Qianglian Huang* and Lanping Zhu
}

"Correspondence:

qlhmath@yahoo.com.cn

College of Mathematics, Yangzhou

University, Yangzhou, 225002, China

\begin{abstract}
In this paper, several weak and strong convergence theorems are established for a new modified iteration with errors for a finite family of nonself mappings which are asymptotically nonexpansive in the intermediate sense in Banach spaces. Mann-type, Ishikawa-type and Noor-type iterations are covered by this new iteration scheme. Our convergence theorems improve, unify and generalize many important results in the current literature.

MSC: $47 \mathrm{H} 10 ; 47 \mathrm{HO} 0 ; 46 \mathrm{~B} 2 \mathrm{O}$

Keywords: asymptotically nonexpansive mapping in the intermediate sense; Kadec-Klee property; Opial's condition; uniformly convex Banach spaces; common fixed points
\end{abstract}

\section{Introduction}

Fixed-point iteration processes for nonexpansive and asymptotically nonexpansive mappings including Mann-type and Ishikawa-type iterations have been studied extensively by many authors (see [1-23] and the references cited therein). In 1991, Schu [14] considered the modified Mann iteration process for an asymptotically nonexpansive map. Later, Tan and $\mathrm{Xu}$ [16] studied the modified Ishikawa iteration process for an asymptotically nonexpansive map. Noor, in 2000, introduced a three-step iterative scheme and studied the approximate solutions of variational inclusion in Hilbert spaces [11]. Later, Cho et al. [4], $\mathrm{Xu}$ and Noor [18] studied weak and strong convergence theorems for the three-step Noor iterations with errors for asymptotically nonexpansive mappings in a uniformly convex Banach space which satisfies Opial's condition or whose norm is Fréchet differentiable. Takahashi and Tamura [15], Shahzad [24] dealt with the iterative scheme for a pair of nonexpansive and asymptotically nonexpansive mappings in a uniformly convex Banach space. In 2006, Plubtieng et al. [12] studied a class of three-step iterative scheme, for three asymptotically nonexpansive mappings, in a uniformly convex Banach space satisfying Opial's condition. In 2007, Fukhar-ud-dina and Khan [5] studied the scheme for three nonexpansive mappings in a uniformly convex Banach space which has Opial's condition or which has a Fréchet differentiable norm or whose dual space has the Kadec-Klee property. Also in 2007, Chidume and Bashir Ali [1] introduced the iterative scheme for a finite family of asymptotically nonexpansive mappings and obtained the weak and strong convergence theorems in a Banach space whose dual space satisfies the Kadec-Klee property. 
In most of these papers, the map $T$ has been assumed to map $C$ into itself. If, however, $C$ is a proper subset of a Banach space $X$ and $T$ maps $C$ into $X$ (as is the case in many applications), then $\left\{x_{n}\right\}$ may not be well defined. One method that has been used to overcome this is to introduce a retraction $P: X \rightarrow C$. Recent results on the approximation of fixed points of nonexpansive and asymptotically nonexpansive nonself mappings can be found in [24-33] and references contained therein.

In 2003, Chidume et al. [2] introduced the following modified Mann iteration process and got the weak and strong convergence theorems for an asymptotically nonexpansive nonself mapping:

$$
x_{1} \in C, \quad x_{n+1}=P\left[\alpha_{n} x_{n}+\left(1-\alpha_{n}\right) T(P T)^{n-1} x_{n}\right], \quad n \geq 1 .
$$

Recently, Wang [32] generalized the above iteration process as follows: $x_{1} \in C$,

$$
\begin{aligned}
& x_{n+1}=P\left[\alpha_{n} x_{n}+\left(1-\alpha_{n}\right) T_{1}\left(P T_{1}\right)^{n-1} y_{n}\right] \\
& y_{n}=P\left[\beta_{n} x_{n}+\left(1-\beta_{n}\right) T_{2}\left(P T_{2}\right)^{n-1} x_{n}\right] .
\end{aligned}
$$

In 2007, Chidume and Bashir Ali [27] introduced the following iteration process for a finite family of asymptotically nonexpansive nonself mappings: $x_{1} \in C$ and

$$
\begin{aligned}
& x_{n+1}=P\left[\alpha_{1 n} x_{n}+\left(1-\alpha_{1 n}\right) T_{1}\left(P T_{1}\right)^{n-1} y_{n+m-2}\right] \\
& y_{n+m-2}=P\left[\alpha_{2 n} x_{n}+\left(1-\alpha_{2 n}\right) T_{2}\left(P T_{2}\right)^{n-1} y_{n+m-3}\right] \\
& \ldots \\
& y_{n}=P\left[\alpha_{m n} x_{n}+\left(1-\alpha_{m n}\right) T_{m}\left(P T_{m}\right)^{n-1} x_{n}\right] .
\end{aligned}
$$

They proved strong convergence theorems in uniformly convex Banach spaces and gave the weak convergence theorem in uniformly convex Banach spaces that satisfy Opial's condition or have a Fréchet differentiable norm. They also gave the weak convergence theorem for nonexpansive nonself mappings in uniformly convex Banach spaces whose dual spaces have the Kadec-Klee property (see [27]).

The concept of asymptotically nonexpansive nonself mappings in the intermediate sense was introduced by Chidume et al. [28] as an important generalization of asymptotically nonexpansive self-mappings in the intermediate sense.

Definition 1.1 Let $C$ be a nonempty subset of a Banach space $X$. Let $P: X \rightarrow C$ be a nonexpansive retraction of $X$ onto $C$. A nonself mapping $T: C \rightarrow X$ is called asymptotically nonexpansive in the intermediate sense if $T$ is continuous and the following inequality holds:

$$
\limsup _{n \rightarrow+\infty} \sup _{x, y \in C}\left(\left\|T(P T)^{n-1} x-T(P T)^{n-1} y\right\|-\|x-y\|\right) \leq 0 \text {. }
$$

It should be noted that in $[28,31,34]$, an asymptotically nonexpansive mapping in the intermediate sense is required to be uniformly continuous. In Definition 1.1, we assume the 
continuity of $T$ instead of uniform continuity. Chidume et al. [28], Plubtieng and Wangkeeree [31], Kim and Kim [34] gave strong convergence theorems for a uniformly continuous mapping which is asymptotically nonexpansive in the intermediate sense in uniformly convex Banach spaces if the mapping is completely continuous. Also, Chidume et al. [28] gave the weak convergence theorem for such a mapping in a uniformly convex Banach space whose dual space has the Kadec-Klee property. However, as we know, it remains open whether the weak convergence theorem of a multi-step iteration process with errors for a finite family of continuous nonself mappings which are asymptotically nonexpansive in the intermediate sense holds in a uniformly convex Banach space which satisfies Opial's condition or whose dual space has the Kadec-Klee property. Since the asymptotically nonexpansive mappings in the intermediate sense are non-Lipschitzian and Bruck's lemma [35] does not extend beyond Lipschitzian mappings, new techniques are needed for this more general case. It is our purpose in this paper to study the following iteration process with errors for approximating common fixed points of a finite family of nonself mappings which are asymptotically nonexpansive in the intermediate sense:

$$
\begin{aligned}
& x_{1} \in C ; \\
& x_{n+1}=P\left[\alpha_{n}^{(1)} x_{n}+\beta_{n}^{(1)} T_{1}\left(P T_{1}\right)^{n-1} y_{n}^{(N-2)}+\gamma_{n}^{(1)} u_{n}^{(1)}\right] ; \\
& y_{n}^{(N-2)}=P\left[\alpha_{n}^{(2)} x_{n}+\beta_{n}^{(2)} T_{2}\left(P T_{2}\right)^{n-1} y_{n}^{(N-3)}+\gamma_{n}^{(2)} u_{n}^{(2)}\right] ; \\
& \ldots \\
& y_{n}^{(1)}=P\left[\alpha_{n}^{(N-1)} x_{n}+\beta_{n}^{(N-1)} T_{N-1}\left(P T_{N-1}\right)^{n-1} y_{n}^{(0)}+\gamma_{n}^{(N-1)} u_{n}^{(N-1)}\right] ; \\
& y_{n}^{(0)}=P\left[\alpha_{n}^{(N)} x_{n}+\beta_{n}^{(N)} T_{N}\left(P T_{N}\right)^{n-1} x_{n}+\gamma_{n}^{(N)} u_{n}^{(N)}\right] .
\end{aligned}
$$

In Section 3, using the technique established in [33], we first give some weak convergence theorems of the iterative scheme (1.1) for a finite family of nonself mappings which are asymptotically nonexpansive in the intermediate sense in a uniformly convex Banach space which satisfies Opial's condition or whose dual space has the Kadec-Klee property. We also establish some strong convergence theorems if one member of the finite family of mappings satisfies a condition weaker than complete continuity. Our results extend and improve the recently announced ones $[1,2,4,5,7,9,12,15,16,18,24,27,28,31,32,34]$ and many others.

\section{Preliminaries}

Let $C$ be a nonempty closed convex subset of a Banach space $X$. Recall that a Banach space $X$ is said to be uniformly convex if, for each $\varepsilon \in[0,2)$, the modulus of convexity of $X$ given by

$$
\delta(\varepsilon)=\inf \left\{1-\frac{1}{2}\|x+y\|:\|x\| \leq 1,\|y\| \leq 1,\|x-y\| \geq \varepsilon\right\}
$$

satisfies the inequality $\delta(\varepsilon)>0$ for all $\varepsilon>0$. We say that $X$ has the Kadec-Klee property if, for every sequence $\left\{x_{n}\right\} \subset X$, whenever $x_{n} \rightarrow x$ with $\left\|x_{n}\right\| \rightarrow\|x\|$, it follows that $x_{n} \rightarrow x$. We would like to remark that a reflexive Banach space $X$ with a Fréchet differentiable norm implies that its dual $X^{*}$ has the Kadec-Klee property, while the converse implication fails [36]. 
Recall that a Banach space $X$ is said to satisfy Opial's condition [37] if $x_{n} \rightarrow x$ and $x \neq y$ implies that

$$
\limsup _{n \rightarrow+\infty}\left\|x_{n}-x\right\|<\limsup _{n \rightarrow+\infty}\left\|x_{n}-y\right\|
$$

A subset $C$ of $X$ is said to be a retract if there exists a continuous mapping $P: X \rightarrow C$ such that $P x=x$ for all $x \in C$. Every closed convex subset of a uniformly convex Banach space is a retract. A mapping $P: X \rightarrow X$ is said to be a retraction if $P^{2}=P$. It follows that if a map $P$ is a retraction, then $P y=y$ for all $y$ in the range of $P$.

Lemma 2.1 [17] Let the nonnegative number sequences $\left\{c_{n}\right\}$ and $\left\{w_{n}\right\}$ satisfy

$$
c_{n+1} \leq c_{n}+w_{n}, \quad n=1,2, \ldots
$$

If $\sum_{n=1}^{+\infty} w_{n}<+\infty$, then $\lim _{n \rightarrow+\infty} c_{n}$ exists.

Lemma 2.2 [14] Suppose that $X$ is a uniformly convex Banach space and for all positive integers $n, 0<p \leq t_{n} \leq q<1$. If $\left\{x_{n}\right\}$ and $\left\{y_{n}\right\}$ are two sequences of $X$ such that $\lim \sup _{n \rightarrow+\infty}\left\|x_{n}\right\| \leq r, \limsup _{n \rightarrow+\infty}\left\|y_{n}\right\| \leq r$ and

$$
\lim _{n \rightarrow+\infty}\left\|t_{n} x_{n}+\left(1-t_{n}\right) y_{n}\right\|=r
$$

hold for some $r \geq 0$. Then $\lim _{n \rightarrow+\infty}\left\|x_{n}-y_{n}\right\|=0$.

Lemma 2.3 [6] Let $X$ be a uniformly convex Banach space. If $\|x\| \leq 1,\|y\| \leq 1$ and $\| x-$ $y \| \geq \varepsilon>0$, then for all $\lambda \in[0,1]$,

$$
\|\lambda x+(1-\lambda) y\| \leq 1-2 \lambda(1-\lambda) \delta(\varepsilon)
$$

Lemma 2.4 (Demiclosedness principle for a nonself-map [38]) Let $C$ be a nonempty closed convex subset of a uniformly convex Banach space $X$ and let $T: C \rightarrow X$ be a nonself mapping which is continuous and asymptotically nonexpansive in the intermediate sense. If $\left\{x_{n}\right\}$ is a sequence in $C$ converging weakly to $x$ and

$$
\lim _{m \rightarrow+\infty} \limsup _{n \rightarrow+\infty}\left\|x_{n}-T(P T)^{m-1} x_{n}\right\|=0,
$$

then $x \in F(T)$, i.e., $T x=x$.

Lemma 2.5 [36] Let $X$ be a reflexive Banach space whose dual $X^{*}$ has the Kadec-Klee property. Let $\left\{x_{n}\right\}$ be a bounded sequence in $X$ and $f, g \in \omega_{\omega}\left(\left\{x_{n}\right\}\right)$. Suppose that

$$
h(\lambda)=\lim _{n \rightarrow+\infty}\left\|\lambda x_{n}+(1-\lambda) f-g\right\|
$$

exists for all $\lambda \in[0,1]$, then $f=g$, where $\omega_{\omega}\left(\left\{x_{n}\right\}\right)$ denotes the set of weak limit points of $\left\{x_{n}\right\}$, i.e., $\omega_{\omega}\left(\left\{x_{n}\right\}\right)=\left\{p \in X\right.$ : there exists a subsequence $\left\{x_{n_{i}}\right\} \subset\left\{x_{n}\right\}$ such that $\left.x_{n_{i}} \rightarrow p\right\}$. 


\section{Main results}

In this section, let $X$ be a uniformly convex Banach space and let $C$ be a nonempty closed convex subset of $X$. Let $P: X \rightarrow C$ be a nonexpansive retraction from $X$ onto $C$. Let $T_{1}, T_{2}, \ldots, T_{N}: C \rightarrow X$ be a finite family of continuous nonself mappings which are asymptotically nonexpansive in the intermediate sense, then we can suppose that

$$
r_{n}=\max \left\{0, \sup _{x, y \in C}\left(\left\|T_{i}\left(P T_{i}\right)^{n-1} x-T_{i}\left(P T_{i}\right)^{n-1} y\right\|-\|x-y\|\right)\right\}, \quad i=1,2, \ldots, N .
$$

Hence $r_{n} \geq 0, \lim _{n \rightarrow+\infty} r_{n}=0$ and for all $x, y \in C$,

$$
\left\|T_{i}\left(P T_{i}\right)^{n-1} x-T_{i}\left(P T_{i}\right)^{n-1} y\right\|-\|x-y\| \leq r_{n}, \quad n \geq 1, i=1,2, \ldots, N .
$$

For a given $x_{1} \in C$, we can define the sequence $\left\{x_{n}\right\} \subset C$ by

$$
\begin{aligned}
& x_{n+1}=P\left[\alpha_{n}^{(1)} x_{n}+\beta_{n}^{(1)} T_{1}\left(P T_{1}\right)^{n-1} y_{n}^{(N-2)}+\gamma_{n}^{(1)} u_{n}^{(1)}\right] ; \\
& y_{n}^{(N-2)}=P\left[\alpha_{n}^{(2)} x_{n}+\beta_{n}^{(2)} T_{2}\left(P T_{2}\right)^{n-1} y_{n}^{(N-3)}+\gamma_{n}^{(2)} u_{n}^{(2)}\right] ; \\
& \ldots \\
& y_{n}^{(1)}=P\left[\alpha_{n}^{(N-1)} x_{n}+\beta_{n}^{(N-1)} T_{N-1}\left(P T_{N-1}\right)^{n-1} y_{n}^{(0)}+\gamma_{n}^{(N-1)} u_{n}^{(N-1)}\right] ; \\
& y_{n}^{(0)}=P\left[\alpha_{n}^{(N)} x_{n}+\beta_{n}^{(N)} T_{N}\left(P T_{N}\right)^{n-1} x_{n}+\gamma_{n}^{(N)} u_{n}^{(N)}\right],
\end{aligned}
$$

where $\left\{\alpha_{n}^{(i)}\right\},\left\{\beta_{n}^{(i)}\right\},\left\{\gamma_{n}^{(i)}\right\}$ are in $[0,1]$ with $0<p \leq \alpha_{n}^{(i)}, \beta_{n}^{(i)} \leq q<1, \alpha_{n}^{(i)}+\beta_{n}^{(i)}+\gamma_{n}^{(i)}=1$, $\sum_{n=1}^{+\infty} \gamma_{n}^{(i)}<+\infty$ and $\left\{u_{n}^{(i)}\right\}$ are bounded sequences in $X, i=1,2, \ldots, N$.

We start our investigation with the following lemmas, which are preparation for the proofs of the main results of this section. In the following, we always assume that $\sum_{n=1}^{+\infty} r_{n}<$ $+\infty$ and the set of common fixed points of $\left\{T_{1}, T_{2}, \ldots, T_{N}\right\}$ is nonempty, i.e.,

$$
\bigcap_{i=1}^{N} F\left(T_{i}\right)=\bigcap_{i=1}^{N}\left\{x \in C: T_{i} x=x\right\} \neq \emptyset .
$$

\section{Lemma 3.1}

$$
\lim _{n \rightarrow+\infty}\left\|x_{n}-f\right\|=r
$$

exists for each $f \in \bigcap_{i=1}^{N} F\left(T_{i}\right)$.

Proof Let $f \in \bigcap_{i=1}^{N} F\left(T_{i}\right)$. Since $\left\{u_{n}^{(i)}\right\}$ are bounded, we can set

$$
M=\sup \left\{\left\|u_{n}^{(i)}-f\right\|: i=1,2, \ldots, N, n \geq 1\right\}<+\infty .
$$

Then

$$
\begin{aligned}
& \left\|y_{n}^{(0)}-f\right\| \\
& \quad=\left\|P\left[\alpha_{n}^{(N)} x_{n}+\beta_{n}^{(N)} T_{N}\left(P T_{N}\right)^{n-1} x_{n}+\gamma_{n}^{(N)} u_{n}^{(N)}\right]-f\right\|
\end{aligned}
$$




$$
\begin{aligned}
& \leq\left\|\left[\alpha_{n}^{(N)} x_{n}+\beta_{n}^{(N)} T_{N}\left(P T_{N}\right)^{n-1} x_{n}+\gamma_{n}^{(N)} u_{n}^{(N)}\right]-f\right\| \\
& \leq \alpha_{n}^{(N)}\left\|x_{n}-f\right\|+\beta_{n}^{(N)}\left\|T_{N}\left(P T_{N}\right)^{n-1} x_{n}-f\right\|+\gamma_{n}^{(N)}\left\|u_{n}^{(N)}-f\right\| \\
& \leq\left\|x_{n}-f\right\|+r_{n}+M \gamma_{n}^{(N)} .
\end{aligned}
$$

Hence we can get

$$
\begin{aligned}
& \left\|y_{n}^{(1)}-f\right\| \\
& \quad \leq\left\|\left[\alpha_{n}^{(N-1)} x_{n}+\beta_{n}^{(N-1)} T_{N-1}\left(P T_{N-1}\right)^{n-1} y_{n}^{(0)}+\gamma_{n}^{(N-1)} u_{n}^{(N-1)}\right]-f\right\| \\
& \quad \leq \alpha_{n}^{(N-1)}\left\|x_{n}-f\right\|+\beta_{n}^{(N-1)}\left\|T_{N-1}\left(P T_{N-1}\right)^{n-1} y_{n}^{(0)}-f\right\|+\gamma_{n}^{(N-1)}\left\|u_{n}^{(N-1)}-f\right\| \\
& \quad \leq \alpha_{n}^{(N-1)}\left\|x_{n}-f\right\|+\beta_{n}^{(N-1)}\left(\left\|y_{n}^{(0)}-f\right\|+r_{n}\right)+M \gamma_{n}^{(N-1)} \\
& \quad \leq \alpha_{n}^{(N-1)}\left\|x_{n}-f\right\|+\beta_{n}^{(N-1)}\left(\left\|x_{n}-f\right\|+2 r_{n}+M \gamma_{n}^{(N)}\right)+M \gamma_{n}^{(N-1)} \\
& \quad=\left\|x_{n}-f\right\|+2 r_{n}+M\left(\gamma_{n}^{(N)}+\gamma_{n}^{(N-1)}\right)
\end{aligned}
$$

and

$$
\begin{aligned}
& \left\|y_{n}^{(N-2)}-f\right\| \\
& \quad \leq\left\|\left[\alpha_{n}^{(2)} x_{n}+\beta_{n}^{(2)} T_{2}\left(P T_{2}\right)^{n-1} y_{n}^{(N-3)}+\gamma_{n}^{(2)} u_{n}^{(2)}\right]-f\right\| \\
& \quad \leq \alpha_{n}^{(2)}\left\|x_{n}-f\right\|+\beta_{n}^{(2)}\left\|T_{2}\left(P T_{2}\right)^{n-1} y_{n}^{(N-3)}-f\right\|+\gamma_{n}^{(2)}\left\|u_{n}^{(2)}-f\right\| \\
& \quad \leq \alpha_{n}^{(2)}\left\|x_{n}-f\right\|+\beta_{n}^{(2)}\left(\left\|y_{n}^{(N-3)}-f\right\|+r_{n}\right)+M \gamma_{n}^{(2)} \\
& \quad \leq\left\|x_{n}-f\right\|+(N-1) r_{n}+M\left(\gamma_{n}^{(N)}+\cdots+\gamma_{n}^{(3)}+\gamma_{n}^{(2)}\right) .
\end{aligned}
$$

Thus we obtain

$$
\begin{aligned}
& \left\|x_{n+1}-f\right\| \\
& \quad \leq\left\|\left[\alpha_{n}^{(1)} x_{n}+\beta_{n}^{(1)} T_{1}\left(P T_{1}\right)^{n-1} y_{n}^{(N-2)}+\gamma_{n}^{(1)} u_{n}^{(1)}\right]-f\right\| \\
& \quad \leq \alpha_{n}^{(1)}\left\|x_{n}-f\right\|+\beta_{n}^{(1)}\left\|T_{1}\left(P T_{1}\right)^{n-1} y_{n}^{(N-2)}-f\right\|+\gamma_{n}^{(1)}\left\|u_{n}^{(1)}-f\right\| \\
& \quad \leq \alpha_{n}^{(1)}\left\|x_{n}-f\right\|+\beta_{n}^{(1)}\left(\left\|y_{n}^{(N-2)}-f\right\|+r_{n}\right)+M \gamma_{n}^{(1)} \\
& \quad \leq\left\|x_{n}-f\right\|+N r_{n}+M\left(\gamma_{n}^{(N)}+\cdots+\gamma_{n}^{(2)}+\gamma_{n}^{(1)}\right) .
\end{aligned}
$$

Set $w_{n}=N r_{n}+M\left(\gamma_{n}^{(N)}+\gamma_{n}^{(N-1)}+\cdots+\gamma_{n}^{(1)}\right)$, then $\sum_{n=1}^{+\infty} w_{n}<+\infty$ and

$$
\left\|x_{n+1}-f\right\| \leq\left\|x_{n}-f\right\|+w_{n}
$$

By Lemma 2.1, we have that

$$
\lim _{n \rightarrow+\infty}\left\|x_{n}-f\right\|=r
$$

exists. This completes the proof. 


\section{Lemma 3.2}

$$
\lim _{m \rightarrow+\infty} \limsup _{n \rightarrow+\infty}\left\|x_{n}-T_{i}\left(P T_{i}\right)^{m-1} x_{n}\right\|=0, \quad i=1,2, \ldots, N .
$$

Proof By Lemma 3.1, we have that $\lim _{n \rightarrow+\infty}\left\|x_{n}-f\right\|=r$ exists. If $r=0$, then it is obvious to see that the conclusion holds. In the following, we assume that $r>0$. According to (3.2),

(3.3) and (3.4), we can get

$$
\limsup _{n \rightarrow+\infty}\left\|y_{n}^{(j)}-f\right\| \leq r, \quad j=0,1, \ldots, N-2 .
$$

Then, for any $j=0,1, \ldots, N-2$,

$$
\limsup _{n \rightarrow+\infty}\left\|T_{N-1-j}\left(P T_{N-1-j}\right)^{n-1} y_{n}^{(j)}-f\right\| \leq r,
$$

and hence the sequences $\left\{T_{N-1-j}\left(P T_{N-1-j}\right)^{n-1} y_{n}^{(j)}\right\}_{n=1}^{+\infty}$ are bounded. By (3.5), we can obtain

$$
\lim _{n \rightarrow+\infty}\left\|\left[\alpha_{n}^{(1)} x_{n}+\beta_{n}^{(1)} T_{1}\left(P T_{1}\right)^{n-1} y_{n}^{(N-2)}+\gamma_{n}^{(1)} u_{n}^{(1)}\right]-f\right\|=r .
$$

We also can see

$$
\limsup _{n \rightarrow+\infty}\left\|T_{1}\left(P T_{1}\right)^{n-1} y_{n}^{(N-2)}-f+\gamma_{n}^{(1)}\left(u_{n}^{(1)}-x_{n}\right)\right\| \leq r
$$

and

$$
\limsup _{n \rightarrow+\infty}\left\|x_{n}-f+\gamma_{n}^{(1)}\left(u_{n}^{(1)}-x_{n}\right)\right\| \leq r .
$$

It follows from Lemma 2.2 and

$$
\begin{aligned}
r= & \lim _{n \rightarrow+\infty}\left\|\left[\alpha_{n}^{(1)} x_{n}+\beta_{n}^{(1)} T_{1}\left(P T_{1}\right)^{n-1} y_{n}^{(N-2)}+\gamma_{n}^{(1)} u_{n}^{(1)}\right]-f\right\| \\
= & \lim _{n \rightarrow+\infty} \|\left(1-\alpha_{n}^{(1)}\right)\left[T_{1}\left(P T_{1}\right)^{n-1} y_{n}^{(N-2)}-f+\gamma_{n}^{(1)}\left(u_{n}^{(1)}-x_{n}\right)\right] \\
& +\alpha_{n}^{(1)}\left[x_{n}-f+\gamma_{n}^{(1)}\left(u_{n}^{(1)}-x_{n}\right)\right]+\gamma_{n}^{(1)}\left[x_{n}-T_{1}\left(P T_{1}\right)^{n-1} y_{n}^{(N-2)}\right] \| \\
= & \lim _{n \rightarrow+\infty} \|\left(1-\alpha_{n}^{(1)}\right)\left[T_{1}\left(P T_{1}\right)^{n-1} y_{n}^{(N-2)}-f+\gamma_{n}^{(1)}\left(u_{n}^{(1)}-x_{n}\right)\right] \\
& +\alpha_{n}^{(1)}\left[x_{n}-f+\gamma_{n}^{(1)}\left(u_{n}^{(1)}-x_{n}\right)\right] \|
\end{aligned}
$$

that

$$
\lim _{n \rightarrow+\infty}\left\|T_{1}\left(P T_{1}\right)^{n-1} y_{n}^{(N-2)}-x_{n}\right\|=0 .
$$

Combining it with

$$
\begin{aligned}
\left\|x_{n}-f\right\| & \leq\left\|x_{n}-T_{1}\left(P T_{1}\right)^{n-1} y_{n}^{(N-2)}\right\|+\left\|T_{1}\left(P T_{1}\right)^{n-1} y_{n}^{(N-2)}-f\right\| \\
& \leq\left\|x_{n}-T_{1}\left(P T_{1}\right)^{n-1} y_{n}^{(N-2)}\right\|+\left\|y_{n}^{(N-2)}-f\right\|+r_{n},
\end{aligned}
$$


we obtain

$$
\liminf _{n \rightarrow+\infty}\left\|y_{n}^{(N-2)}-f\right\| \geq r
$$

Thus $\lim _{n \rightarrow+\infty}\left\|y_{n}^{(N-2)}-f\right\|=r$, according to (3.4), we have

$$
\begin{aligned}
r= & \lim _{n \rightarrow+\infty}\left\|\left[\alpha_{n}^{(2)} x_{n}+\beta_{n}^{(2)} T_{2}\left(P T_{2}\right)^{n-1} y_{n}^{(N-3)}+\gamma_{n}^{(2)} u_{n}^{(2)}\right]-f\right\| \\
= & \lim _{n \rightarrow+\infty} \|\left(1-\alpha_{n}^{(2)}\right)\left[T_{2}\left(P T_{2}\right)^{n-1} y_{n}^{(N-3)}-f+\gamma_{n}^{(2)}\left(u_{n}^{(2)}-x_{n}\right)\right] \\
& +\alpha_{n}^{(2)}\left[x_{n}-f+\gamma_{n}^{(2)}\left(u_{n}^{(2)}-x_{n}\right)\right]+\gamma_{n}^{(2)}\left[x_{n}-T_{2}\left(P T_{2}\right)^{n-1} y_{n}^{(N-3)}\right] \| \\
= & \lim _{n \rightarrow+\infty} \|\left(1-\alpha_{n}^{(2)}\right)\left[T_{2}\left(P T_{2}\right)^{n-1} y_{n}^{(N-3)}-f+\gamma_{n}^{(2)}\left(u_{n}^{(2)}-x_{n}\right)\right] \\
& +\alpha_{n}^{(2)}\left[x_{n}-f+\gamma_{n}^{(2)}\left(u_{n}^{(2)}-x_{n}\right)\right] \| .
\end{aligned}
$$

Noting

$$
\limsup _{n \rightarrow+\infty}\left\|T_{2}\left(P T_{2}\right)^{n-1} y_{n}^{(N-3)}-f+\gamma_{n}^{(2)}\left(u_{n}^{(2)}-x_{n}\right)\right\| \leq r
$$

and

$$
\limsup _{n \rightarrow+\infty}\left\|x_{n}-f+\gamma_{n}^{(2)}\left(u_{n}^{(2)}-x_{n}\right)\right\| \leq r
$$

by Lemma 2.2 again, we have

$$
\lim _{n \rightarrow+\infty}\left\|T_{2}\left(P T_{2}\right)^{n-1} y_{n}^{(N-3)}-x_{n}\right\|=0 .
$$

Similarly, we can get

$$
\begin{aligned}
\lim _{n \rightarrow+\infty}\left\|T_{3}\left(P T_{3}\right)^{n-1} y_{n}^{(N-4)}-x_{n}\right\| & =\cdots=\lim _{n \rightarrow+\infty}\left\|T_{N-1}\left(P T_{N-1}\right)^{n-1} y_{n}^{(0)}-x_{n}\right\| \\
& =\lim _{n \rightarrow+\infty}\left\|T_{N}\left(P T_{N}\right)^{n-1} x_{n}-x_{n}\right\|=0 .
\end{aligned}
$$

Therefore, by (3.1) and (3.7),

$$
\lim _{n \rightarrow+\infty}\left\|x_{n+1}-x_{n}\right\| \leq \lim _{n \rightarrow+\infty}\left\|\beta_{n}^{(1)}\left[T_{1}\left(P T_{1}\right)^{n-1} y_{n}^{(N-2)}-x_{n}\right]+\gamma_{n}^{(1)}\left(u_{n}^{(1)}-x_{n}\right)\right\|=0
$$

and similarly, we can have

$$
\lim _{n \rightarrow+\infty}\left\|y_{n}^{(N-2)}-x_{n}\right\|=\cdots=\lim _{n \rightarrow+\infty}\left\|y_{n}^{(1)}-x_{n}\right\|=\lim _{n \rightarrow+\infty}\left\|y_{n}^{(0)}-x_{n}\right\|=0 .
$$

It follows from the inequality (3.7) and

$$
\begin{aligned}
& \left\|T_{1}\left(P T_{1}\right)^{n-1} x_{n}-x_{n}\right\| \\
& \quad \leq\left\|T_{1}\left(P T_{1}\right)^{n-1} x_{n}-T_{1}\left(P T_{1}\right)^{n-1} y_{n}^{(N-2)}\right\|+\left\|T_{1}\left(P T_{1}\right)^{n-1} y_{n}^{(N-2)}-x_{n}\right\| \\
& \quad \leq\left\|x_{n}-y_{n}^{(N-2)}\right\|+r_{n}+\left\|T_{1}\left(P T_{1}\right)^{n-1} y_{n}^{(N-2)}-x_{n}\right\|
\end{aligned}
$$


that

$$
\lim _{n \rightarrow+\infty}\left\|T_{1}\left(P T_{1}\right)^{n-1} x_{n}-x_{n}\right\|=0
$$

Thus, for any fixed $m$, we have $\lim _{n \rightarrow+\infty}\left\|x_{n}-x_{n+m}\right\|=0$ and

$$
\begin{aligned}
\| x_{n}- & T_{1}\left(P T_{1}\right)^{m-1} x_{n} \| \\
\leq & \left\|x_{n}-x_{n+m}\right\|+\left\|x_{n+m}-T_{1}\left(P T_{1}\right)^{n+m-1} x_{n+m}\right\| \\
& \quad\left\|T_{1}\left(P T_{1}\right)^{n+m-1} x_{n+m}-T_{1}\left(P T_{1}\right)^{n+m-1} x_{n}\right\|+\left\|T_{1}\left(P T_{1}\right)^{n+m-1} x_{n}-T_{1}\left(P T_{1}\right)^{m-1} x_{n}\right\| \\
& \leq 2\left\|x_{n}-x_{n+m}\right\|+\left\|x_{n+m}-T_{1}\left(P T_{1}\right)^{n+m-1} x_{n+m}\right\|+r_{n+m}+\left\|\left(P T_{1}\right)^{n} x_{n}-x_{n}\right\|+r_{m} \\
\leq & 2\left\|x_{n}-x_{n+m}\right\|+\left\|x_{n+m}-T_{1}\left(P T_{1}\right)^{n+m-1} x_{n+m}\right\|+r_{n+m}+\left\|T_{1}\left(P T_{1}\right)^{n-1} x_{n}-x_{n}\right\|+r_{m} .
\end{aligned}
$$

This implies

$$
\limsup _{n \rightarrow+\infty}\left\|x_{n}-T_{1}\left(P T_{1}\right)^{m-1} x_{n}\right\| \leq r_{m}
$$

Therefore,

$$
\lim _{m \rightarrow+\infty} \limsup _{n \rightarrow+\infty}\left\|x_{n}-T_{1}\left(P T_{1}\right)^{m-1} x_{n}\right\|=0 .
$$

Noting (3.8) and

$$
\begin{aligned}
& \left\|T_{2}\left(P T_{2}\right)^{n-1} x_{n}-x_{n}\right\| \\
& \quad \leq\left\|T_{2}\left(P T_{2}\right)^{n-1} x_{n}-T_{2}\left(P T_{2}\right)^{n-1} y_{n}^{(N-3)}\right\|+\left\|T_{2}\left(P T_{2}\right)^{n-1} y_{n}^{(N-3)}-x_{n}\right\| \\
& \quad \leq\left\|x_{n}-y_{n}^{(N-3)}\right\|+r_{n}+\left\|T_{2}\left(P T_{2}\right)^{n-1} y_{n}^{(N-3)}-x_{n}\right\|,
\end{aligned}
$$

we can see

$$
\lim _{n \rightarrow+\infty}\left\|T_{2}\left(P T_{2}\right)^{n-1} x_{n}-x_{n}\right\|=0
$$

Thus, for any fixed $m$,

$$
\begin{aligned}
\| x_{n}- & T_{2}\left(P T_{2}\right)^{m-1} x_{n} \| \\
\leq & \left\|x_{n}-x_{n+m}\right\|+\left\|x_{n+m}-T_{2}\left(P T_{2}\right)^{n+m-1} x_{n+m}\right\| \\
& \quad\left\|T_{2}\left(P T_{2}\right)^{n+m-1} x_{n+m}-T_{2}\left(P T_{2}\right)^{n+m-1} x_{n}\right\|+\left\|T_{2}\left(P T_{2}\right)^{n+m-1} x_{n}-T_{2}\left(P T_{2}\right)^{m-1} x_{n}\right\| \\
& \leq 2\left\|x_{n}-x_{n+m}\right\|+\left\|x_{n+m}-T_{2}\left(P T_{2}\right)^{n+m-1} x_{n+m}\right\|+r_{n+m}+\left\|\left(P T_{2}\right)^{n} x_{n}-x_{n}\right\|+r_{m} \\
\leq & 2\left\|x_{n}-x_{n+m}\right\|+\left\|x_{n+m}-T_{2}\left(P T_{2}\right)^{n+m-1} x_{n+m}\right\|+r_{n+m}+\left\|T_{2}\left(P T_{2}\right)^{n-1} x_{n}-x_{n}\right\|+r_{m},
\end{aligned}
$$

which means

$$
\limsup _{n \rightarrow+\infty}\left\|x_{n}-T_{2}\left(P T_{2}\right)^{m-1} x_{n}\right\| \leq r_{m} .
$$


Therefore,

$$
\lim _{m \rightarrow+\infty} \limsup _{n \rightarrow+\infty}\left\|x_{n}-T_{2}\left(P T_{2}\right)^{m-1} x_{n}\right\|=0 .
$$

By the same argument, we can get

$$
\lim _{n \rightarrow+\infty}\left\|x_{n}-T_{3}\left(P T_{3}\right)^{m-1} x_{n}\right\|=\cdots=\lim _{n \rightarrow+\infty}\left\|x_{n}-T_{N}\left(P T_{N}\right)^{m-1} x_{n}\right\|=0 .
$$

This completes the proof.

Define the operator $W_{n}: C \rightarrow C$ by

$$
\begin{aligned}
& W_{n} x=P\left[\alpha_{n}^{(1)} x+\beta_{n}^{(1)} T_{1}\left(P T_{1}\right)^{n-1} x^{(N-2)}+\gamma_{n}^{(1)} u_{n}^{(1)}\right] \\
& x^{(N-2)}=P\left[\alpha_{n}^{(2)} x+\beta_{n}^{(2)} T_{2}\left(P T_{2}\right)^{n-1} x^{(N-3)}+\gamma_{n}^{(2)} u_{n}^{(2)}\right] ; \\
& \cdots \\
& x^{(1)}=P\left[\alpha_{n}^{(N-1)} x+\beta_{n}^{(N-1)} T_{N-1}\left(P T_{N-1}\right)^{n-1} x^{(0)}+\gamma_{n}^{(N-1)} u_{n}^{(N-1)}\right] ; \\
& x^{(0)}=P\left[\alpha_{n}^{(N)} x+\beta_{n}^{(N)} T_{N}\left(P T_{N}\right)^{n-1} x+\gamma_{n}^{(N)} u_{n}^{(N)}\right],
\end{aligned}
$$

where $x \in C$. Then $x_{n+1}=W_{n} x_{n}$ and for all $x, y \in C$, we have

$$
\begin{aligned}
\left\|x^{(0)}-y^{(0)}\right\| & \leq \alpha_{n}^{(N)}\|x-y\|+\beta_{n}^{(N)}\left\|T_{N}\left(P T_{N}\right)^{n-1} x-T_{N}\left(P T_{N}\right)^{n-1} y\right\| \\
& \leq\|x-y\|+r_{n}, \\
\left\|x^{(1)}-y^{(1)}\right\| & \leq \alpha_{n}^{(N-1)}\|x-y\|+\beta_{n}^{(N-1)}\left\|T_{N-1}\left(P T_{N-1}\right)^{n-1} x^{(0)}-T_{N-1}\left(P T_{N-1}\right)^{n-1} y^{(0)}\right\| \\
& \leq \alpha_{n}^{(N-1)}\|x-y\|+\beta_{n}^{(N-1)}\left(\left\|x^{(0)}-y^{(0)}\right\|+r_{n}\right) \\
& \leq\|x-y\|+2 r_{n},
\end{aligned}
$$

and

$$
\begin{aligned}
\left\|W_{n} x-W_{n} y\right\| & \leq \alpha_{n}^{(1)}\|x-y\|+\beta_{n}^{(1)}\left\|T_{1}\left(P T_{1}\right)^{n-1} x^{(N-2)}-T_{1}\left(P T_{1}\right)^{n-1} y^{(N-2)}\right\| \\
& \leq \alpha_{n}^{(1)}\|x-y\|+\beta_{n}^{(1)}\left(\left\|x^{(N-2)}-y^{(N-2)}\right\|+r_{n}\right) \\
& \leq \alpha_{n}^{(1)}\|x-y\|+\beta_{n}^{(1)}\left(\|x-y\|+N r_{n}\right) \\
& \leq\|x-y\|+w_{n} .
\end{aligned}
$$

For any $f \in \bigcap_{i=1}^{N} F\left(T_{i}\right)$, we get

$$
\begin{aligned}
\left\|f^{(0)}-f\right\| & \leq\left\|\alpha_{n}^{(N)} f+\beta_{n}^{(N)} T_{N}\left(P T_{N}\right)^{n-1} f+\gamma_{n}^{(N)} u_{n}^{(N)}-f\right\| \\
& =\gamma_{n}^{(N)}\left\|u_{n}^{(N)}-f\right\| \leq M \gamma_{n}^{(N)}, \\
\left\|f^{(1)}-f\right\| & \leq \beta_{n}^{(N-1)}\left\|f^{(0)}-f\right\|+r_{n}+\gamma_{n}^{(N-1)}\left\|u_{n}^{(N-1)}-f\right\| \\
& \leq r_{n}+M\left(\gamma_{n}^{(N)}+\gamma_{n}^{(N-1)}\right),
\end{aligned}
$$




$$
\left\|f^{(N-2)}-f\right\| \leq(N-2) r_{n}+M\left(\gamma_{n}^{(N)}+\cdots+\gamma_{n}^{(2)}\right)
$$

and

$$
\begin{aligned}
\left\|W_{n} f-f\right\| & \leq \beta_{n}^{(1)}\left\|f^{(N-2)}-f\right\|+r_{n}+\gamma_{n}^{(1)}\left\|u_{n}^{(1)}-f\right\| \\
& \leq(N-1) r_{n}+M\left(\gamma_{n}^{(N)}+\cdots+\gamma_{n}^{(1)}\right) \leq w_{n} .
\end{aligned}
$$

Set $S_{n, m}=W_{n+m-1} W_{n+m-2} \cdots W_{n+1} W_{n}: C \rightarrow C$, then $x_{n+m}=S_{n, m} x_{n}$ and for any $x, y \in C$,

$$
\left\|S_{n, m} x-S_{n, m} y\right\| \leq\|x-y\|+\left(w_{n+m-1}+\cdots+w_{n+1}+w_{n}\right) .
$$

We also need the following lemma, which plays a crucial role in dealing with the case of the iteration with errors. It is easy to see $S_{n, m} f \equiv f$ if $\gamma_{n}^{(i)} \equiv 0$ for all $i=1,2, \ldots, N$ and all $n \geq 1$.

\section{Lemma 3.3}

$$
\lim _{n \rightarrow+\infty} \limsup _{m \rightarrow+\infty}\left\|S_{n, m} f-f\right\|=0, \quad \forall f \in \bigcap_{i=1}^{N} F\left(T_{i}\right) .
$$

Proof By (3.10), (3.11) and

$$
\begin{aligned}
& \begin{aligned}
\left\|S_{n, 2} f-f\right\| & =\left\|W_{n+1} W_{n} f-f\right\| \\
& \leq\left\|W_{n+1} W_{n} f-W_{n+1} f\right\|+\left\|W_{n+1} f-f\right\| \\
& \leq\left\|W_{n} f-f\right\|+w_{n+1}+w_{n+1} \\
& \leq w_{n}+2 w_{n+1},
\end{aligned} \\
& \begin{array}{l}
\| S_{n, m-1} f-f
\end{array} \\
& \quad \leq w_{n}+2 w_{n+1}+\cdots+2 w_{n+m-2},
\end{aligned}
$$

we get

$$
\begin{aligned}
\left\|S_{n, m} f-f\right\| & =\left\|W_{n+m-1} S_{n, m-1} f-f\right\| \\
& \leq\left\|W_{n+m-1} S_{n, m-1} f-W_{n+m-1} f\right\|+\left\|W_{n+m-1} f-f\right\| \\
& \leq\left\|S_{n, m-1} f-f\right\|+w_{n+m-1}+w_{n+m-1} \\
& \leq w_{n}+2 w_{n+1}+\cdots+2 w_{n+m-1} \\
& \leq 2\left(w_{n}+w_{n+1}+\cdots+w_{n+m-1}\right) .
\end{aligned}
$$

Then fixing $n$ and taking the limsup for $m$, we obtain

$$
\limsup _{m \rightarrow+\infty}\left\|S_{n, m} f-f\right\| \leq 2 \sum_{i=n}^{+\infty} w_{i}
$$


Thus,

$$
\lim _{n \rightarrow+\infty} \limsup _{m \rightarrow+\infty}\left\|S_{n, m} f-f\right\| \leq \lim _{n \rightarrow+\infty} 2 \sum_{i=n}^{+\infty} w_{i}=0 .
$$

This completes the proof.

Lemma 3.4 Let $f, g \in \bigcap_{i=1}^{N} F\left(T_{i}\right)$ and $\lambda \in[0,1]$, then

$$
h(\lambda)=\lim _{n \rightarrow+\infty}\left\|\lambda x_{n}+(1-\lambda) f-g\right\|
$$

exists.

Proof It follows from Lemma 3.1 that $r=\lim _{n \rightarrow+\infty}\left\|x_{n}-f\right\|$ exists. If $\lambda=0,1$ or $r=0$, then the conclusion holds. In the following, we assume that $r>0$ and $\lambda \in(0,1)$. Then, for any $\varepsilon>0$, there exists $d>0(d<\varepsilon)$ such that

$$
(r+d)\left[1-2 \lambda(1-\lambda) \delta\left(\frac{\varepsilon}{r+d}\right)\right]<r-d,
$$

where $\delta$ is the modulus of convexity of the norm. Hence there exists a positive integer $n_{0}$ such that for all $n>n_{0}$,

$$
r-\frac{d}{4} \leq\left\|x_{n}-f\right\| \leq r+\frac{d}{4}
$$

and

$$
\sum_{i=n}^{+\infty} w_{i} \leq \lambda(1-\lambda) \frac{d}{4}<\frac{\varepsilon}{4}
$$

Now we claim that for all $n>n_{0}$,

$$
\left\|S_{n, m}\left[\lambda x_{n}+(1-\lambda) f\right]-\left[\lambda S_{n, m} x_{n}+(1-\lambda) S_{n, m} f\right]\right\| \leq \varepsilon, \quad \forall m=1,2, \ldots
$$

Otherwise, we can suppose that

$$
\left\|S_{n, m}\left[\lambda x_{n}+(1-\lambda) f\right]-\left[\lambda S_{n, m} x_{n}+(1-\lambda) S_{n, m} f\right]\right\| \geq \varepsilon
$$

for some $m$. Put $z=\lambda x_{n}+(1-\lambda) f, x=(1-\lambda)\left(S_{n, m} z-S_{n, m} f\right)$ and $y=\lambda\left(S_{n, m} x_{n}-S_{n, m} z\right)$, then by (3.12), (3.14) and (3.15),

$$
\begin{aligned}
\|x\| & \leq(1-\lambda)\left(\left\|S_{n, m} z-S_{n, m} f\right\|\right) \\
& \leq(1-\lambda)\left[\|z-f\|+\left(w_{n+m-1}+\cdots+w_{n+1}+w_{n}\right)\right] \\
& \leq \lambda(1-\lambda)\left(\left\|x_{n}-f\right\|+\frac{d}{4}\right) \\
& \leq \lambda(1-\lambda)(r+d)
\end{aligned}
$$


and

$$
\begin{aligned}
\|y\| & =\lambda\left\|S_{n, m} x_{n}-S_{n, m} z\right\| \\
& \leq \lambda\left[\left\|x_{n}-z\right\|+\left(w_{n+m-1}+\cdots+w_{n+1}+w_{n}\right)\right] \\
& \leq \lambda(1-\lambda)\left(\left\|x_{n}-f\right\|+\frac{d}{4}\right) \\
& \leq \lambda(1-\lambda)(r+d) .
\end{aligned}
$$

We also have

$$
\|x-y\|=\left\|S_{n, m}\left[\lambda x_{n}+(1-\lambda) f\right]-\left[\lambda S_{n, m} x_{n}+(1-\lambda) S_{n, m} f\right]\right\| \geq \varepsilon
$$

and

$$
\lambda x+(1-\lambda) y=\lambda(1-\lambda)\left(S_{n, m} x_{n}-S_{n, m} f\right) .
$$

So, by using Lemma 2.3, we get

$$
\begin{aligned}
& \lambda(1-\lambda)\left\|S_{n, m} x_{n}-S_{n, m} f\right\| \\
& \quad=\|\lambda x+(1-\lambda) y\| \\
& \quad \leq \lambda(1-\lambda)(r+d)\left[1-2 \lambda(1-\lambda) \delta\left(\frac{\varepsilon}{\lambda(1-\lambda)(r+d)}\right)\right] \\
& \quad \leq \lambda(1-\lambda)(r+d)\left[1-2 \lambda(1-\lambda) \delta\left(\frac{\varepsilon}{r+d}\right)\right],
\end{aligned}
$$

and then by (3.13), (3.15) and (3.16), we have

$$
\begin{aligned}
r-d & \leq\left\|x_{n+m}-f\right\|-\left\|S_{n, m} f-f\right\| \\
& \leq\left\|S_{n, m} x_{n}-f\right\|-\left\|S_{n, m} f-f\right\| \\
& \leq\left\|S_{n, m} x_{n}-S_{n, m} f\right\| \\
& \leq(r+d)\left[1-2 \lambda(1-\lambda) \delta\left(\frac{\varepsilon}{r+d}\right)\right] .
\end{aligned}
$$

This contradicts (3.14). Thus we can conclude that for all $n>n_{0}$, (3.17) holds. Hence, for all $n>n_{0}$,

$$
\begin{aligned}
\left\|\lambda x_{n+m}+(1-\lambda) f-g\right\| \\
=\left\|\lambda S_{n, m} x_{n}+(1-\lambda) f-g\right\| \\
\leq\left\|\left[\lambda S_{n, m} x_{n}+(1-\lambda) S_{n, m} f\right]-S_{n, m}\left[\lambda x_{n}+(1-\lambda) f\right]\right\|+(1-\lambda)\left\|S_{n, m} f-f\right\| \\
\quad+\left\|S_{n, m}\left[\lambda x_{n}+(1-\lambda) f\right]-S_{n, m} g\right\|+\left\|S_{n, m} g-g\right\| \\
\leq 2 \varepsilon+\left\|S_{n, m} f-f\right\|+\left\|\lambda x_{n}+(1-\lambda) f-g\right\|+\left\|S_{n, m} g-g\right\| .
\end{aligned}
$$


For any fixed $n>n_{0}$, we can take the limsup for $m$ and obtain

$$
\begin{aligned}
& \limsup _{m \rightarrow+\infty}\left\|\lambda x_{m}+(1-\lambda) f-g\right\| \\
& \quad \leq\left\|\lambda x_{n}+(1-\lambda) f-g\right\|+2 \varepsilon+\limsup _{m \rightarrow+\infty}\left\|S_{n, m} f-f\right\|+\limsup _{m \rightarrow+\infty}\left\|S_{n, m} g-g\right\| .
\end{aligned}
$$

Hence we have

$$
\limsup _{m \rightarrow+\infty}\left\|\lambda x_{m}+(1-\lambda) f-g\right\| \leq \liminf _{n \rightarrow+\infty}\left\|\lambda x_{n}+(1-\lambda) f-g\right\|+2 \varepsilon
$$

Since $\varepsilon>0$ is arbitrary, this implies that

$$
h(\lambda)=\lim _{n \rightarrow+\infty}\left\|\lambda x_{n}+(1-\lambda) f-g\right\|
$$

exists. This completes the proof.

Remark 3.1 If the mappings $T_{1}, T_{2}, \ldots, T_{N}$ are asymptotically nonexpansive, we can use Bruck's lemma [35] to prove Lemma 3.4. While Bruck's lemma is not valid for nonLipschitzian mappings, we must introduce some new techniques to establish a similar inequality. As we haven seen, we use mainly the technique of the modulus of convexity and our proof is straightforward.

Now we can prove the weak convergence theorem of the iterative scheme (3.1).

Theorem 3.1 Let $C$ be a nonempty closed convex subset of a uniformly convex Banach space $X$ which satisfies Opial's condition or whose dual $X^{*}$ has the Kadec-Klee property. Let $P: X \rightarrow C$ be a nonexpansive retraction from $X$ onto $C$. Let $T_{1}, T_{2}, \ldots, T_{N}: C \rightarrow X$ be a finite family of nonself mappings which are asymptotically nonexpansive in the intermediate sense with $\bigcap_{i=1}^{N} F\left(T_{i}\right) \neq \emptyset$ and the sequences $\left\{r_{n}\right\} \subset[0,+\infty)$ satisfying $\sum_{n=1}^{+\infty} r_{n}<+\infty$. Let $\left\{x_{n}\right\}$ be defined by

$$
\begin{aligned}
& x_{1} \in C ; \\
& x_{n+1}=P\left[\alpha_{n}^{(1)} x_{n}+\beta_{n}^{(1)} T_{1}\left(P T_{1}\right)^{n-1} y_{n}^{(N-2)}+\gamma_{n}^{(1)} u_{n}^{(1)}\right] ; \\
& y_{n}^{(N-2)}=P\left[\alpha_{n}^{(2)} x_{n}+\beta_{n}^{(2)} T_{2}\left(P T_{2}\right)^{n-1} y_{n}^{(N-3)}+\gamma_{n}^{(2)} u_{n}^{(2)}\right] ; \\
& \cdots \\
& y_{n}^{(1)}=P\left[\alpha_{n}^{(N-1)} x_{n}+\beta_{n}^{(N-1)} T_{N-1}\left(P T_{N-1}\right)^{n-1} y_{n}^{(0)}+\gamma_{n}^{(N-1)} u_{n}^{(N-1)}\right] ; \\
& y_{n}^{(0)}=P\left[\alpha_{n}^{(N)} x_{n}+\beta_{n}^{(N)} T_{N}\left(P T_{N}\right)^{n-1} x_{n}+\gamma_{n}^{(N)} u_{n}^{(N)}\right],
\end{aligned}
$$

where $\left\{\alpha_{n}^{(i)}\right\},\left\{\beta_{n}^{(i)}\right\},\left\{\gamma_{n}^{(i)}\right\}$ are in $[0,1]$ with $0<p \leq \alpha_{n}^{(i)}, \beta_{n}^{(i)} \leq q<1, \alpha_{n}^{(i)}+\beta_{n}^{(i)}+\gamma_{n}^{(i)}=1$, $\sum_{n=1}^{+\infty} \gamma_{n}^{(i)}<+\infty$ and $\left\{u_{n}^{(i)}\right\}$ are bounded sequences in $X, i=1,2, \ldots, N$. Then $\left\{x_{n}\right\}$ converges weakly to a common fixed point of $\left\{T_{i}\right\}_{i=1}^{N}$.

Proof It suffices to prove that the set $\omega_{\omega}\left(\left\{x_{n}\right\}\right)$ is a singleton. Since $X$ is reflexive and $\left\{x_{n}\right\}$ is bounded, we obtain $\omega_{\omega}\left(\left\{x_{n}\right\}\right) \neq \emptyset$. Assuming that $f, g \in \omega_{\omega}\left(\left\{x_{n}\right\}\right)$, in the following, we need 
to show $f=g$. First, by Lemma 2.4 and Lemma 3.2, we know $f, g \in \bigcap_{i=1}^{N} F\left(T_{i}\right)$. Second, on the one hand, if $X^{*}$ has the Kadec-Klee property, then from Lemma 3.4 and Lemma 2.5, we can get $f=g$. On the other hand, if $X$ satisfies Opial's condition, we assume that $f \neq g$ and two subsequences $\left\{x_{n_{i}}\right\}$ and $\left\{x_{n_{j}}\right\}$ in $\left\{x_{n}\right\}$ such that $x_{n_{i}} \rightarrow f$ and $x_{n_{j}} \rightarrow g$. Hence by Opial's condition and Lemma 3.1, we get

$$
\begin{aligned}
\lim _{n \rightarrow+\infty}\left\|x_{n}-f\right\| & =\lim _{i \rightarrow+\infty}\left\|x_{n_{i}}-f\right\| \\
& <\lim _{i \rightarrow+\infty}\left\|x_{n_{i}}-g\right\|=\lim _{n \rightarrow+\infty}\left\|x_{n}-g\right\|=\lim _{j \rightarrow+\infty}\left\|x_{n_{j}}-g\right\| \\
& <\lim _{i \rightarrow+\infty}\left\|x_{n_{j}}-f\right\|=\lim _{n \rightarrow+\infty}\left\|x_{n}-f\right\| .
\end{aligned}
$$

This contraction implies $f=g$. This completes the proof.

Remark 3.2 Theorem 3.1 generalizes and improves many recent important results. For instance, if $N=1$ and $T_{1}: C \rightarrow X$ is a uniformly continuous mapping which is asymptotically nonexpansive in the intermediate sense, then we can get Theorem 3.13 in [28]. If $T_{1}, T_{2}, \ldots, T_{N}: C \rightarrow X$ are asymptotically nonexpansive nonself mappings and (1) $\gamma_{n}^{(i)} \equiv 0$, then we can obtain Theorem 3.6 in [27]; (2) $\gamma_{n}^{(i)} \equiv 0, T_{i}: C \rightarrow C$, then we can get Theorem 3.4 in [1]; (3) $T_{i}: C \rightarrow C$ and $N \leq 3$, then we can get Theorem 3.10 in [2], Theorem 2.1 in [4], Theorems 3.1-3.2 in [7], Theorem 1 in [9], Theorem 2.9 in [12], Theorem 3.3 in [15], Theorems 3.1-3.2 in [16], Theorem 3.5 in [32] and many others.

If $\left\{T_{i}\right\}_{i=1}^{N}$ is a family of nonexpansive mappings, we can have the following theorem, which is an extension of Theorem 4.1 in [5], Theorem 1 in [9], Theorem 3.2 in [15], Theorem 3.5 and Theorem 4.1 in [24], Theorem 3.9 and Theorem 4.2 in [27] and others. The proof is immediate corollaries of our lemmas and Theorem 3.1.

Theorem 3.2 Let $C$ be a nonempty closed convex subset of a uniformly convex Banach space $X$ which satisfies Opial's condition or whose dual $X^{*}$ has the Kadec-Klee property. Let $P: X \rightarrow C$ be a nonexpansive retraction from $X$ onto $C$. Let $T_{1}, T_{2}, \ldots, T_{N}: C \rightarrow X$ be a finite family of nonself nonexpansive mappings with $\bigcap_{i=1}^{N} F\left(T_{i}\right) \neq \emptyset$. Let $\left\{x_{n}\right\}$ be defined by

$$
\begin{aligned}
& x_{1} \in C ; \\
& x_{n+1}=P\left[\alpha_{n}^{(1)} x_{n}+\beta_{n}^{(1)} T_{1} y_{n}^{(N-2)}+\gamma_{n}^{(1)} u_{n}^{(1)}\right] ; \\
& y_{n}^{(N-2)}=P\left[\alpha_{n}^{(2)} x_{n}+\beta_{n}^{(2)} T_{2} y_{n}^{(N-3)}+\gamma_{n}^{(2)} u_{n}^{(2)}\right] ; \\
& \ldots \\
& y_{n}^{(1)}=P\left[\alpha_{n}^{(N-1)} x_{n}+\beta_{n}^{(N-1)} T_{N-1} y_{n}^{(0)}+\gamma_{n}^{(N-1)} u_{n}^{(N-1)}\right] ; \\
& y_{n}^{(0)}=P\left[\alpha_{n}^{(N)} x_{n}+\beta_{n}^{(N)} T_{N} x_{n}+\gamma_{n}^{(N)} u_{n}^{(N)}\right],
\end{aligned}
$$

where $\left\{\alpha_{n}^{(i)}\right\},\left\{\beta_{n}^{(i)}\right\},\left\{\gamma_{n}^{(i)}\right\}$ are in $[0,1]$ with $0<p \leq \alpha_{n}^{(i)}, \beta_{n}^{(i)} \leq q<1, \alpha_{n}^{(i)}+\beta_{n}^{(i)}+\gamma_{n}^{(i)}=1$, $\sum_{n=1}^{+\infty} \gamma_{n}^{(i)}<+\infty$ and $\left\{u_{n}^{(i)}\right\}$ are bounded sequences in $X, i=1,2, \ldots, N$. Then $\left\{x_{n}\right\}$ converges weakly to a common fixed point of $\left\{T_{i}\right\}_{i=1}^{N}$.

Now we can give the strong convergence theorem of the scheme (3.1). 
Theorem 3.3 Let $C$ be a nonempty closed convex subset of a uniformly convex Banach space $X$ and let $P: X \rightarrow C$ be a nonexpansive retraction from $X$ onto $C$. Let $T_{1}, T_{2}, \ldots, T_{N}$ : $C \rightarrow X$ be a finite family of nonself mappings which are asymptotically nonexpansive in the intermediate sense and $\left\{x_{n}\right\}$ be as in Theorem 3.1. Then $\left\{x_{n}\right\}$ converges strongly to a common fixed point of $\left\{T_{i}\right\}_{i=1}^{N}$ if and only if

$$
\lim _{n \rightarrow+\infty} d\left(x_{n}, F\right)=0
$$

where $F$ denotes the set of common fixed points of $\left\{T_{i}\right\}_{i=1}^{N}$, i.e., $F=\bigcap_{i=1}^{N} F\left(T_{i}\right)$.

Proof We only need to show the sufficiency. If $\lim _{n \rightarrow+\infty} d\left(x_{n}, F\right)=0$, then for any $\varepsilon>0$, there exists a positive integer $n_{0}$ such that for all $n \geq n_{0}$,

$$
d\left(x_{n}, F\right)<\frac{\varepsilon}{4} \quad \text { and } \quad \sum_{i=n}^{+\infty} w_{i}<\frac{\varepsilon}{3} .
$$

Hence, for any $n \geq n_{0}$, there exists $f \in F$ such that $\left\|x_{n}-f\right\|<\frac{\varepsilon}{3}$. Therefore, for any $m \in N$, by (3.6),

$$
\begin{aligned}
& \left\|x_{n+m}-x_{n}\right\| \\
& \quad \leq\left\|x_{n+m}-f\right\|+\left\|x_{n}-f\right\| \\
& \quad \leq\left\|x_{n+m-1}-f\right\|+w_{n+m-1}+\left\|x_{n}-f\right\| \\
& \quad \ldots \\
& \quad \leq\left\|x_{n}-f\right\|+\left(w_{n}+\cdots+w_{n+m-2}+w_{n+m-1}\right)+\left\|x_{n}-f\right\| \\
& \quad \leq \frac{\varepsilon}{3}+\frac{\varepsilon}{3}+\frac{\varepsilon}{3}=\varepsilon,
\end{aligned}
$$

which implies that $\left\{x_{n}\right\}$ is a Cauchy sequence in $C$ and then it must converge to some point in $C$. Set $\lim _{n \rightarrow+\infty} x_{n}=p$, since $\lim _{n \rightarrow+\infty} d\left(x_{n}, F\right)=0$ and $F$ is closed, we get $p \in F$. This completes the proof.

In the following, we shall give a sufficient condition to ensure the strong convergence of the iterative sequence (3.1). We need the following notions. Recall that a finite family of nonself mappings $T_{1}, T_{2}, \ldots, T_{N}: C \rightarrow X$ with $F=\bigcap_{i=1}^{N} F\left(T_{i}\right) \neq \emptyset$ satisfies Condition $(\widetilde{C})$ if there exists a nondecreasing function $f:[0,+\infty) \rightarrow[0,+\infty)$ with $f(0)=0, f(r)>0$, for $r \in(0,+\infty)$, such that at least one of the $\left\{T_{i}\right\}_{i=1}^{N}$ satisfies condition $(\widetilde{I})$, i.e.,

$$
\left\|T_{i} x-x\right\| \geq f(d(x, F)), \quad \forall x \in C,
$$

for at least one $T_{i}, 1 \leq i \leq N$, where $d(x, F)=\inf \{\|x-p\|: p \in F\}$.

A mapping $T: C \rightarrow X$ is said to be demicompact if, for any bounded sequence $\left\{x_{n}\right\}$ in $C$ such that $x_{n}-T x_{n}$ converges, there exists a subsequence, say $\left\{x_{n_{j}}\right\}$ of $\left\{x_{n}\right\}$, such that $\left\{x_{n_{j}}\right\}$ converges strongly to some point in $C$. $T$ is said to be completely continuous if it is continuous and for every bounded sequence $\left\{x_{n}\right\}$, there exists a subsequence, say $\left\{x_{n_{j}}\right\}$ of $\left\{x_{n}\right\}$, such that the sequence $T x_{n_{j}}$ converges to some element of the range of $T$. 
It is well known that every continuous and demicompact mapping must satisfy condition $(\widetilde{I})$ since every completely continuous mapping $T: C \rightarrow C$ is continuous and demicompact so that it satisfies condition $(\widetilde{I})$. Therefore, the condition $(\widetilde{I})$ is weaker than the demicompactness and complete continuity (see [27]). Next we shall give several strong convergence theorems in uniformly convex Banach spaces if one member of the finite family of asymptotically nonexpansive in the intermediate sense mappings $\left\{T_{i}\right\}_{i=1}^{N}$ satisfies condition $\widetilde{I})$.

Theorem 3.4 Let $C$ be a nonempty closed convex subset of a uniformly convex Banach space $X$ and let $P: X \rightarrow C$ be a nonexpansive retraction from $X$ onto C. Let $T_{1}, T_{2}, \ldots, T_{N}$ : $C \rightarrow X$ be a finite family of uniformly continuous nonself mappings which are asymptotically nonexpansive in the intermediate sense and let $\left\{x_{n}\right\}$ be as in Theorem 3.1. If the family $\left\{T_{1}, T_{2}, \ldots, T_{N}\right\}$ satisfies condition $(\widetilde{C})$, then $\left\{x_{n}\right\}$ converges strongly to a common fixed point of $\left\{T_{i}\right\}_{i=1}^{N}$.

Proof Without loss of generality, we assume that $T_{1}$ satisfies condition $(\widetilde{I})$, i.e.,

$$
\left\|T_{1} x-x\right\| \geq f(d(x, F)), \quad \forall x \in C .
$$

Hence we have

$$
\left\|T_{1} x_{n}-x_{n}\right\| \geq f\left(d\left(x_{n}, F\right)\right), \quad n=1,2, \ldots
$$

Then by (3.9), the uniform continuity of $T_{1}$ and

$$
\begin{aligned}
& \left\|x_{n}-T_{1} x_{n}\right\| \\
& \quad \leq\left\|x_{n}-x_{n+1}\right\|+\left\|x_{n+1}-T_{1}\left(P T_{1}\right)^{n} x_{n+1}\right\| \\
& \quad+\left\|T_{1}\left(P T_{1}\right)^{n} x_{n+1}-T_{1}\left(P T_{1}\right)^{n} x_{n}\right\|+\left\|T_{1}\left(P T_{1}\right)^{n} x_{n}-T_{1} x_{n}\right\| \\
& \quad \leq 2\left\|x_{n}-x_{n+1}\right\|+\left\|x_{n+1}-T_{1}\left(P T_{1}\right)^{n} x_{n+1}\right\|+\left\|T_{1}\left(P T_{1}\right)^{n} x_{n}-T_{1} x_{n}\right\|+r_{n+1},
\end{aligned}
$$

we derive

$$
\lim _{n \rightarrow+\infty}\left\|x_{n}-T_{1} x_{n}\right\|=0
$$

By (3.6), we have for all $f \in F,\left\|x_{n+1}-f\right\| \leq\left\|x_{n}-f\right\|+w_{n}$, where $\sum_{n=1}^{+\infty} w_{n}<+\infty$. Hence

$$
d\left(x_{n+1}, F\right) \leq d\left(x_{n}, F\right)+w_{n}
$$

Then it follows from Lemma 2.1 that $\lim _{n \rightarrow+\infty} d\left(x_{n}, F\right)$ exists. Hence, by (3.18) and (3.19), we see $\lim _{n \rightarrow+\infty} f\left(d\left(x_{n}, F\right)\right)=0$ and therefore,

$$
\lim _{n \rightarrow+\infty} d\left(x_{n}, F\right)=0
$$

By Theorem 3.3, we can get what we desired. This completes the proof. 
Remark 3.3 From Theorem 3.4, we can get Theorem 3.8 and Theorem 3.10 in [28], Theorem 3.5 in [31], Theorem 1 and Theorem 2 in [34].

For completeness, we conclude with the following strong convergence theorem for a finite family of nonexpansive and asymptotically nonexpansive nonself mappings.

Theorem 3.5 Let $C$ be a nonempty closed convex subset of a uniformly convex Banach space $X$ and let $P: X \rightarrow C$ be a nonexpansive retraction from $X$ onto C. Let $T_{1}, T_{2}, \ldots, T_{N}$ : $C \rightarrow X$ be a finite family of asymptotically nonexpansive nonself mappings and $\left\{x_{n}\right\}$ be as in Theorem 3.1. If the family $\left\{T_{1}, T_{2}, \ldots, T_{N}\right\}$ satisfies condition $(\widetilde{C})$, then $\left\{x_{n}\right\}$ converges strongly to a common fixed point of $\left\{T_{i}\right\}_{i=1}^{N}$.

Theorem 3.6 Let $C$ be a nonempty closed convex subset of a uniformly convex Banach space $X$ and let $P: X \rightarrow C$ be a nonexpansive retraction from $X$ onto $C$. Let $T_{1}, T_{2}, \ldots, T_{N}$ : $C \rightarrow X$ be a finite family of nonexpansive nonself mappings and $\left\{x_{n}\right\}$ be as in Theorem 3.2. If the family $\left\{T_{1}, T_{2}, \ldots, T_{N}\right\}$ satisfies condition $(\widetilde{C})$, then $\left\{x_{n}\right\}$ converges strongly to a common fixed point of $\left\{T_{i}\right\}_{i=1}^{N}$.

Remark 3.4 Theorem 3.5 and Theorem 3.6 generalize and improve many recent important results such as Theorem 3.5 in [1], Theorem 3.7 in [2], Theorem 2.4 in [4], Theorem 4.2 in [5], Theorem 2 in [9], Theorem 2.4 in [12], Theorems 2.1-2.3 in [18], Theorem 3.6 in [24], Theorem 3.4 and Theorem 4.1 in [27], Theorems 3.3-3.4 in [32] and others.

Competing interests

The authors declare that they have no competing interests.

Authors' contributions

All authors contributed equally and significantly in writing this paper. All authors read and approved the final manuscript.

\section{Acknowledgements}

The authors are grateful to the referees for their constructive comments and helpful suggestions.

This research is supported by the National Science Foundation of China (11201410, 11271316 and 11101353), the Natural Science Foundation of Jiangsu Province (BK2012260) and the Natural Science Foundation of Jiangsu Education

Committee (10KJB110012 and 11KJB110018).

Received: 16 March 2013 Accepted: 8 June 2013 Published: 1 July 2013

\section{References}

1. Chidume, CE, Ali, B: Weak and strong convergence theorems for finite families of asymptotically nonexpansive mappings in Banach spaces. J. Math. Anal. Appl. 330, 377-387 (2007)

2. Chidume, CE, Ofoedu, EU, Zegeye, H: Strong and weak convergence theorems for asymptotically nonexpansive mappings. J. Math. Anal. Appl. 280, 364-374 (2003)

3. Chidume, CE, Chidume, CO: Iterative methods for common fixed points for a countable family of nonexpansive mappings in uniformly convex spaces. Nonlinear Anal. 71,4346-4356 (2009)

4. Cho, YJ, Zhou, HY, Guo, G: Weak and strong convergence theorems for three-step iterations with errors for asymptotically nonexpansive mappings. Comput. Math. Appl. 47, 707-717 (2004)

5. Fukhar-ud-din, $\mathrm{H}$, Khan, AR: Approximating common fixed points of asymptotically nonexpansive maps in uniformly convex Banach spaces. Comput. Math. Appl. 53, 1349-1360 (2007)

6. Groetsch, CW: A note on segmenting Mann iterates. J. Math. Anal. Appl. 40, 369-372 (1972)

7. Guo, W, Guo, W: Weak convergence theorems for asymptotically nonexpansive nonself-mappings. Appl. Math. Lett. 24, 2181-2185 (2011)

8. Ishikawa, S: Fixed points by a new iteration method. Proc. Am. Math. Soc. 44, 147-150 (1974)

9. Khan, SH, Fukhar-ud-din, H: Weak and strong convergence of a scheme with errors for two nonexpansive mappings. Nonlinear Anal. 61, 1295-1301 (2005)

10. Mann, WR: Mean value methods in iteration. Proc. Am. Math. Soc. 44, 506-510 (1953)

11. Noor, MA: New approximation schemes for general variational inequalities. J. Math. Anal. Appl. 251, $217-229$ (2000) 
12. Plubtieng, S, Wangkeeree, R, Punpaeng, R: On the convergence of modified Noor iterations with errors for asymptotically nonexpansive mappings. J. Math. Anal. Appl. 322, 1018-1029 (2006)

13. Plubtieng, S, Ungchittrakool, K, Wangkeeree, R: Implicit iterations of two finite families for nonexpansive mappings in Banach spaces. Numer. Funct. Anal. Optim. 28, 737-749 (2007)

14. Schu, J: Weak and strong convergence to fixed points of asymptotically nonexpansive mappings. Bull. Aust. Math. Soc. 43, 153-159 (1991)

15. Takahashi, W, Tamura, T: Convergence theorems for a pair of nonexpansive mappings. J. Convex Anal. 5, 45-56 (1998)

16. Tan, KK, Xu, HK: Fixed point iteration processes for asymptotically nonexpansive mapping. Proc. Am. Math. Soc. 122, 733-739 (1994)

17. Tan, KK, Xu, HK: Approximating fixed points of nonexpansive mappings by the Ishikawa iteration process. J. Math. Anal. Appl. 178, 301-308 (1993)

18. Xu, BL, Noor, MA: Fixed-points iteration for asymptotically nonexpansive mappings in Banach spaces. J. Math. Anal. Appl. 267, 444-453 (2002)

19. Xu, HK: Existence and convergence for fixed points of mappings of asymptotically nonexpansive type. Nonlinear Anal. 16, 1139-1146 (1991)

20. Yao, YH, Chen, RD, Yao, JC: Strong convergence and certain control conditions for modified Mann iteration. Nonlinear Anal. 68, 1687-1693 (2008)

21. Zegeye, $\mathrm{H}$, Shahzad, $\mathrm{N}$ : Approximation methods for a common fixed point of a finite family of nonexpansive mappings. Numer. Funct. Anal. Optim. 28, 1405-1419 (2007)

22. Zhou, YY, Chang, SS: Convergence of implicit iteration process for a finite family of asymptotically nonexpansive mappings in Banach spaces. Numer. Funct. Anal. Optim. 23, 911-921 (2002)

23. Zhou, HY, Cho, YJ, Kang, SM: A new iterative algorithm for approximating common fixed points for asymptotically nonexpansive mappings. Fixed Point Theory Appl. 2007, Article ID 64974 (2007)

24. Shahzad, N: Approximating fixed points of non-self nonexpansive mappings in Banach spaces. Nonlinear Anal. 61, 1031-1039 (2005)

25. Chen, LC, Yao, JC: Strong convergence of an iterative algorithm for nonself multimaps in Banach spaces. Nonlinear Anal. 71, 4476-4485 (2009)

26. Chen, W, Guo, W: Convergence theorems for two finite families of asymptotically nonexpansive mappings. Math. Comput. Model. 54, 1311-1319 (2011)

27. Chidume, CE, Ali, B: Approximation of common fixed points for finite families of nonself asymptotically nonexpansive mappings in Banach spaces. J. Math. Anal. Appl. 326, 960-973 (2007)

28. Chidume, CE, Shahzad, N, Zegeye, H: Convergence theorems for mappings which are asymptotically nonexpansive in the intermediate sense. Numer. Funct. Anal. Optim. 25, 239-257 (2004)

29. Guo, W, Cho, YJ, Guo, W: Convergence theorems for mixed type asymptotically nonexpansive mappings. Fixed Point Theory Appl. 2012, 224 (2012)

30. Pathak, HK, Cho, YJ, Kang, SM: Strong and weak convergence theorems for nonself-asymptotically perturbed nonexpansive mappings. Nonlinear Anal. 70, 1929-1938 (2009)

31. Plubtieng, S, Wangkeeree, R: Strong convergence theorems for three-step iterations with errors for non-Lipschitzian nonself-mappings in Banach spaces. Comput. Math. Appl. 51, 1093-1102 (2006)

32. Wang, L: Strong and weak convergence theorems for common fixed points of nonself asymptotically nonexpansive mappings. J. Math. Anal. Appl. 323, 550-557 (2006)

33. Zhu, LP, Huang, QL, Li, G: Weak convergence theorem for the three-step iterations of non-Lipschitzian nonself mappings in Banach spaces. Fixed Point Theory Appl. 2011, 106 (2011)

34. Kim, GE, Kim, TH: Mann and Ishikawa iterations with errors for non-Lipschitzian mappings in Banach spaces. Comput. Math. Appl. 42, 1565-1570 (2001)

35. Bruck, RE: A simple proof of the mean ergodic theorem for nonlinear contractions in Banach spaces. Isr. J. Math. 32, 107-116 (1979)

36. Kaczor, W: Weak convergence of almost orbits of asymptotically nonexpansive commutative semigroups. J. Math. Anal. Appl. 272, 565-574 (2002)

37. Opial, Z: Weak convergence of the sequence of successive approximations for nonexpansive mappings. Bull. Am. Math. Soc. 73, 591-597 (1967)

38. Kaczor, W: A nonstandard proof of a generalized demiclosedness principle. Ann. Univ. Mariae Curie-SkJodowska, Sect. A LIX, 43-50 (2005)

doi:10.1186/1687-1812-2013-170

Cite this article as: Huang and Zhu: Weak and strong convergence theorems for a finite family of non-Lipschitzian nonself mappings in Banach spaces. Fixed Point Theory and Applications 2013 2013:170. 